\title{
Estratégias, ações e políticas no cuidado à pessoa idosa: um olhar da gestão municipal
}

\author{
Strategies, actions and policies in care for elderly people: a view of municipal management \\ Estrategias, acciones y políticas para el cuidado de personas mayores: una visión de la \\ gestión municipal
}

Natalia Raiane Silva Vieira ${ }^{1 *}$, Maria Alzira Rêgo Pinheiro ${ }^{2}$, Ana Elza Oliveira de Mendonça ${ }^{2}$, Angela Maria de Medeiros Soares², Jônia Cybele Santos Lima², Vilani Medeiros de Araújo Nunes².

\section{RESUMO}

Objetivo: Identificar o conhecimento dos gestores municipais a respeito das políticas de saúde direcionadas à pessoa idosa, caracterizar os profissionais que atuam na gestão municipal e descrever os serviços oferecidos de Atenção à Pessoa Idosa nos municípios estudados. Métodos: Estudo transversal, analítico, descritivo e de abordagem qualitativa, realizado junto aos gestores da atenção básica de três municípios da região metropolitana do Estado do Rio Grande do Norte. Foi aplicado um roteiro com questões previamente elaboradas no contexto da temática do estudo e aplicado por meio da técnica da entrevista. Utilizou-se análise descritiva e frequência absoluta e relativa. Resultados: Os municípios possuem avanços quanto aos programas, serviços e espaços de convivência voltados a pessoa idosa. Quanto a acessibilidade ainda há desafios a serem sanados, grande parte dos serviços não possuem critérios para se tornarem acessíveis aos idosos. A percepção dos gestores investigada no estudo quanto as políticas de saúde voltadas a pessoa idosa, aponta para familiaridade com as portarias, documentos e práticas que norteiam o cuidado no envelhecimento. Conclusão: Os gestores fundamentais para o processo de mudança do modo de produção de saúde, sendo assim devem provocar mudanças no processo de saúde e doença em busca de uma qualidade de vida para a população.

Palavras-chave: Gestão em saúde, Envelhecimento, Saúde do idoso, Política pública.

\begin{abstract}
Objective: To identify the knowledge of municipal managers regarding health policies aimed at the elderly, to characterize the professionals who work in municipal management and to describe the services offered to Elderly Care in the municipalities studied. Methods: Cross-sectional, analytical, descriptive study with a qualitative approach, carried out with primary care managers in the three cities in the metropolitan region of the State of Rio Grande do Norte. A script was applied with questions previously elaborated in the context of the study theme and applied through the interview technique. Descriptive analysis and absolute and relative frequency were used. Results: The municipalities have made progress in terms of programs, services and living spaces for the elderly. As for accessibility, there are still challenges to be solved, most services do not have criteria to make them accessible to the elderly. The perception of managers investigated in the study regarding health policies aimed at the elderly, points to familiarity with the ordinances, documents and practices that guide care in aging. Conclusion: Managers are fundamental in the process of changing the mode of health production, so they must cause changes in the health and disease process in search of a quality of life for the population.
\end{abstract}

Key word: Health management, Aging, Health of the elderly, Public policy.

1 Secretaria do Estado de Saúde Pública (SESAP). Natal - RN. *E-mail: nataliaravieira@hotmail.com

2 Universidade Federal do Rio Grande do Norte (UFRN), Natal - RN.

SUBMETIDO EM: 8/2020

ACEITO EM: 10/2020

PUBLICADO EM: 11/2020

REAS/EJCH | Vol.12(11) | e4948 | DOI: https://doi.org/10.25248/reas.e4948.2020 Página 1 de 9 


\section{RESUMEN}

Objetivo: Identificar el conocimiento de los administradores municipales sobre las políticas de salud dirigidas a las personas mayores, caracterizar a los profesionales que trabajan en la gestión municipal y describir los servicios que se ofrecen a los ancianos en los municipios estudiados. Métodos: Estudio descriptivo, analítico, transversal, con enfoque cualitativo, realizado con gestores de atención primaria en tres municipios de la región metropolitana del Estado de Rio Grande do Norte. Se aplicó un guión con preguntas previamente elaboradas en el contexto del tema de estudio y aplicadas con la técnica de la entrevista. Se utilizó el análisis descriptivo y la frecuencia absoluta y relativa. Resultados: Los municipios han avanzado en términos de programas, servicios y espacios de vida para personas mayores. En cuanto a la accesibilidad, todavía hay desafíos por resolver, la mayoría de los servicios no tienen criterios para hacerlos accesibles a las personas mayores. La percepción de los gerentes investigados en el estudio con respecto a las políticas de salud dirigidas a los ancianos, apunta a la familiaridad con las ordenanzas, documentos y prácticas que guían la atención en el envejecimiento. Conclusión: Los gerentes son fundamentales en el proceso de cambiar el modo de producción de salud, por lo que deben causar cambios en el proceso de salud y enfermedad en busca de una calidad de vida para la población.

Palabras clave: Gestión en salud, Envejecimiento, Salud del anciano, Política pública.

\section{INTRODUÇÃO}

Em decorrência do desenvolvimento tecnológico e científico, da velocidade da transição demográfica e da redução das taxas de natalidade e mortalidade, observou-se, na sociedade moderna, um aumento da expectativa de vida. Tal fato possibilitou que o processo de envelhecimento humano caminhasse em ritmo acelerado no mundo todo (MENDES JLV, et al., 2018).

Em 2017, a população brasileira era composta por 207.1 milhões de habitantes, sendo $14,6 \%$ a participação de pessoas da terceira idade, o que compreende mais de 30 milhões de pessoas. Reconhecese um país como estruturalmente envelhecido, quando sua população alcança, no mínimo, $7 \%$ de pessoas com 60 anos ou mais. Sendo assim, o Brasil já pode ser considerado um país de pessoas idosas. Dessa forma, políticas públicas, ações e estratégias de saúde voltadas para o idoso devem ser estruturadas, implementadas e reforçadas (CASTRO JLC, et al., 2020).

No tocante à garantia dos direitos da pessoa idosa, a Constituição Federal de 1988 possibilitou a responsabilização da família, do Estado e da sociedade no cuidado e proteção dessa população, permitindo a seguridade dos seus direitos sociais, culturais, previdenciários e familiares. Além disso, a partir da elaboração do Estatuto do Idoso, por meio da Lei no 10.741 de 1 de outubro de 2003, foram acrescentados outros direitos, como o atendimento prioritário quanto à políticas públicas, assistência social e benefícios fornecidos pelo Estado (MIRANDA EC, et al., 2014).

Nesse sentido, historicamente, os avanços das ações e políticas públicas de atenção à pessoa idosa são recentes. Foi iniciada em 1994 com a Política Nacional do Idoso, responsável por assegurar direitos e criar condições para a promoção da autonomia, integração e participação efetiva na sociedade. Em 2006, surgiu a Política Nacional de Saúde da Pessoa Idosa, primeira política brasileira relacionada à pessoa idosa (MOURA LKB, et al., 2020; SILVA JLBV, et al., 2020).

O Pacto pela Saúde também trouxe sua importância na priorização da saúde do idoso, compartilhado entre as três esferas de governo, instalado no ano de 2006. Em 2007, ocorreu a distribuição da primeira Caderneta de Saúde da Pessoa Idosa (CSPI) utilizada para conhecimento multidimensional do idoso. Em seguida, sucedeu-se o compromisso nacional para o envelhecimento humano ativo, concretizada por meio do decreto no 8.114, de 30 de setembro de 2013, que objetiva reunir esforços para valorização, promoção e defesa dos direitos da pessoa idosa. E, por fim, mais recentemente, a Política Nacional de Atenção Básica compreendeu os idosos em suas ações de promoção, prevenção e proteção (BALIEIRO VSL, et al., 2020; GOMES CBS, et al., 2020). 
No panorama atual percebe-se a ausência de sistematização na implantação de políticas públicas que direcionem e sejam voltadas para atenção à saúde da pessoa idosa na saúde pública nos seus níveis de atenção. Diante disso, os gestores de diferentes instâncias necessitam instituir políticas que proporcionem condições favoráveis para conscientizar a população do envelhecimento saudável com convivência familiar e coletiva (PEREIRA KCR, et al., 2017).

Consequentemente, há importância da implantação de serviços e programas que incorporem a saúde da pessoa idosa em sua formação. Assim, a inquietude quanto às questões, ações, políticas e estratégias de cuidado da pessoa idosa motivaram a realização do presente estudo visando proporcionar contribuições para a gestão municipal. Dessa forma, este estudo tem como objetivos: identificar o conhecimento dos gestores municipais a respeito das políticas de saúde direcionadas a pessoa idosa, caracterizar os profissionais que atuam na gestão municipal e descrever os serviços oferecidos de Atenção à Pessoa Idosa nos municípios estudados.

\section{MÉTODOS}

Trata-se de um estudo transversal, analítico, descritivo e de abordagem quantitativa. Inicialmente, foram identificados os municípios de realização do estudo verificando as ações e os avanços relacionados às políticas de saúde da pessoa idosa.

O presente estudo foi realizado em três municípios da região metropolitana do Estado do Rio Grande do Norte que participaram das oficinas de implantação da caderneta de saúde da pessoa idosa. Participaram do estudo os gestores municipais de saúde de cada município, de acordo com a disponibilidade (secretário municipal de saúde, coordenadores da atenção básica e coordenadores da saúde do idoso). Sendo assim, a amostra da pesquisa foi composta por seis gestores.

Em alguns municípios, a gestão era composta por secretaria de saúde e coordenador de atenção básica e não possuía o profissional coordenador de saúde do idoso. Apenas em um município existia o profissional da área técnica de saúde da pessoa idosa. Foram selecionados os municípios da região metropolitana por apresentarem proximidade com a capital do Estado e possuírem a região administrativa centralizada na secretaria do Estado.

As variáveis identificadas no processo do estudo e posterior análise foram: sexo, idade, formação, tempo de formação, instituição de formação, município, titulação, curso de formação complementar, ano de formação, temas de políticas de saúde do idoso abordados nos cursos, políticas de saúde do idoso que obtém o conhecimento, conhecimento sobre a Caderneta de Saúde da Pessoa Idosa, implementada no município, capacitações para implantação da caderneta do idoso, forma de implantação da caderneta, presença de planejamento para implantação, existência de manuais que orientam a política de saúde da pessoa idosa, existência de capacitações no município na área de envelhecimento humano, acessibilidade, presença de acessibilidade nas unidades para atendimento aos idosos, os serviços de atenção a pessoa idosa no município e a existência de programa de atenção domiciliar para idosos, serviços de saúde que compõem a rede de atenção à saúde da pessoa idosa e o conhecimento do gestor quanto as políticas de saúde da pessoa idosa e as ações desenvolvidas no município para garantia do cuidado.

Foram utilizadas como base as diretrizes operacionais do pacto pela saúde $2006 \mathrm{com}$ a portaria do Ministério da Saúde (MS) no 399, publicada em 22 de fevereiro de 2006, a nova Política Nacional de Atenção Básica (PNAB), Portaria ํo 2.436 de 21 de setembro de 2017 e o caderno de Atenção Básica ํo19Envelhecimento e Saúde da Pessoa Idosa (BRASIL, 2006). A coleta foi realizada junto a profissionais que aceitaram participar após leitura e informações descritas no Termo de Consentimento Livre e Esclarecido (TCLE).

Foram enviados ofícios aos gestores dos três municípios que coordenavam a Atenção Básica, dos quais obteve-se o aceite e confirmação de agendamento da entrevista. Antes de comparecer ao serviço de saúde, a pesquisadora confirmou a disponibilidade do gestor na data e horário agendados pelas secretarias municipais de saúde de cada município.

REAS/EJCH | Vol.12(11) | e4948 | DOI: https://doi.org/10.25248/reas.e4948.2020 Página 3 de 9 
O instrumento de coleta de dados foi desenvolvido pela pesquisadora e constou de duas partes: a primeira referente aos dados de caracterização dos gestores e a segunda composta por questões abertas referente as políticas de saúde do idoso.

As entrevistas foram realizadas de segunda a sexta-feira no turno matutino, seguindo o agendamento. A sala utilizada para entrevista de forma individualizada foi definida por cada gestor, assegurando a privacidade e confidencialidade das respostas. As entrevistas foram gravadas e tiveram duração média de 30 minutos. É importante destacar que antes de iniciar as gravações os participantes foram esclarecidos e assinaram termo específico aprovado pelo comitê de ética em pesquisa. Os participantes da pesquisa foram devidamente esclarecidos sobre o procedimento de coleta de dados e convidados a formalizar sua participação por meio da leitura e assinatura do Termo de Consentimento Livre e Esclarecido (TCLE).

Este foi preenchido conforme determina o Conselho Nacional de Saúde de acordo com a Resolução № 466/2012 que trata sobre pesquisas envolvendo seres humanos. A pesquisa foi aprovada pelo Comitê de Ética em Pesquisa do Hospital Universitário Onofre Lopes, com Certificado de Apresentação de Apreciação Ética (CAAE) ㄲo 17232919.3.0000.5292.

Antes da realização da pesquisa os gestores assinaram a carta de anuência permitindo a realização da pesquisa em seus estabelecimentos. A coleta de dados se deu em uma etapa nos meses de agosto a setembro de 2019. Os dados foram coletados em formulário elaborado pelos pesquisadores após uma revisão da literatura. A análise estatística dos dados será realizada a partir de análise descritiva das variáveis de estudo, mediante a distribuição de frequência absoluta e relativa para as variáveis categóricas.

\section{RESULTADOS E DISCUSSÃO}

Participaram do estudo seis gestores atuantes nos municípios da região metropolitana do Rio Grande do Norte, sendo uma secretária de saúde, duas coordenadoras de atenção básica, duas secretárias adjuntas de saúde e uma referência técnica em saúde do idoso, com faixa etária de 33 a 67 anos, mediana de 45,5 anos, $100 \%$ do sexo feminino e $66,7 \%$ com graduação em Enfermagem. As outras profissões que tiveram destaque no estudo foram Odontologia 16,6\% e Serviço Social 16,65.

Damasceno CKCS, et al. (2016) reforçam que a Enfermagem é a categoria mais presente para gestão e gerenciamento, pois utiliza ferramentas com foco na descentralização administrativa, criatividade, comunicação informal, liderança e resolutividade nos conflitos. Semelhantemente, no estudo realizado no Paraná por Ohira RHK, et al. (2014), avaliou o perfil dos gerentes atuantes nos serviços de Atenção Básica e evidenciou que $91,1 \%$ dos participantes são do sexo feminino e com idades que variaram entre 21 a 62 anos, com mediana de 30 anos. A categoria profissional predominante dos gerentes era de enfermeiros com $83,3 \%$.

As instituições de formação dos gestores referiam-se a $83,1 \%$ pertencentes à Instituições Privadas e $16,6 \%$ à Instituições Públicas. Todos os profissionais possuem especialização $100 \%$. Dentre estes, 33,3\% fizeram duas especializações, 16,6\% fizeram uma especialização e 16,6\% concluíram quatro especializações. Para o tipo de especialização $71,4 \%$ são da área de Saúde Pública e $28,5 \%$ são especializações voltadas para a clínica das profissões com objetivo de aprimoramento de técnicas

Os cargos exercidos encontrados na pesquisa foram: Secretaria de Saúde Adjunta (33,3\%), Coordenação de Programas da Atenção Básica (16,6\%), Coordenação de Atenção Básica (16,6\%), Referência Técnica do Núcleo da Pessoa Idosa $(16,6 \%)$ e Secretária de Saúde (16,6\%). O tempo de formação variou entre 10 a 20 anos, representando $66,6 \%$, de 20 a 30 anos, com $16,6 \%$ e de 40 a 50 anos, $16,6 \%$. Os gestores, em grande parte $(83,3 \%)$, realizaram cursos complementares na área de gestão e destes $(66,6 \%)$ foi oferecido por uma Universidade Pública do Estado nos anos 2000 (16,6\%), em 2010 (33,3\%), em 2013 (16,6\%) e em 2014 $(16,6 \%)$. Nota-se que, neste estudo, grande parte das gestoras realizou um curso complementar na área de gestão tornando-as qualificadas para desempenhar tal função.

Os cursos complementares na área de gestão auxiliam para garantia do cuidado integral e na tomada de decisões, sendo importantes para o processo de formação e na composição de uma gestão qualificada. As diferentes temáticas devem ser abordadas nos cursos para que o gestor amplie o conhecimento nas 
diferentes áreas do cuidado para que ações não direcionem apenas em áreas especificas do cuidado (TREVISO P, et al., 2017).

Durante o envelhecimento, o gestor deve atentar para o cuidado de forma integral. Dessa forma, apenas $16,6 \%$ dos gestores tiveram, em seus cursos complementares de gestão, disciplinas que abordaram políticas de saúde da pessoa idosa. Para Camargo SM, et al. (2016), é imprescindível a capacitação e atualização dos profissionais que estão no cuidado direto à pessoa idosa para assim promover o autocuidado com interdependência, sempre respeitando a sua autonomia, como também preservar, manter e recuperar a capacidade funcional da pessoa idosa. Foram abordadas questões acerca do conhecimento dos gestores quanto às políticas públicas voltadas para saúde da pessoa idosa (Tabela 1).

Tabela 1 - Políticas e instrumentos de saúde da pessoa idosa de conhecimento dos gestores, 2019.

\begin{tabular}{lc}
\hline Políticas e instrumentos voltados para pessoa idosa de conhecimento dos gestores & Proporção \\
\hline Caderneta de Saúde da Pessoa Idosa & $100 \%$ \\
Política Nacional de Atenção Básica & $100 \%$ \\
Política Nacional de Saúde da Pessoa Idosa & $100 \%$ \\
Política Nacional do Idoso & $83,3 \%$ \\
Estatuto do Idoso & $50 \%$ \\
Pacto pela Saúde & - \\
Portaria SAS no 1771 de 21 de novembro de 2017 & - \\
Outros & $66,6 \%$ \\
\hline
\end{tabular}

Legenda: $($ - ) = não foi citado.

Fonte: VIEIRA NRS, et al., 2020.

As políticas mais lembradas entre os gestores durante a entrevista foram: Política Nacional de Saúde da Pessoa Idosa (100\%), Política Nacional de Atenção Básica (100\%), Caderneta de Saúde da Pessoa Idosa $(100 \%)$ e Política Nacional do Idoso (83,3\%). Os instrumentos mais mencionados por gestores recordam o papel que eles possuem na atenção à saúde da pessoa idosa, presentes, diariamente, na rotina dos processos de trabalho das equipes de saúde e dos gestores. As políticas são relembradas para reforçar o processo de cuidado dessa faixa etária.

Neste sentido, a Política Nacional de Saúde da Pessoa Idosa apresenta medidas de saúde de acordo com os princípios e diretrizes do SUS para promover o autocuidado e independência dos idosos. Para Ferreira VHS, et al. (2020) e Piuvezam G, et al. (2016), a PNAB, recentemente alterada, situa a saúde do idoso como uma das principais áreas do cuidado das Equipes de Saúde da Família e dos equipamentos que compõe a Atenção Básica.

Em seguimento, tem-se a Caderneta de Saúde da Pessoa Idosa como instrumento norteador para equipes de saúde na atuação do cuidado à Pessoa Idosa, com foco na qualificação da atenção a saúde. De acordo com Ramos LV, et al. (2019) e Paulino LF, et al. (2017) a Política Nacional do Idoso foi uma das primeiras políticas a surgirem para assegurar os direitos e deveres dos idosos e aparece como um instrumento para incentivar a autonomia e participação ativa na sociedade como um espaço de respeito e reconhecimento.

O Pacto pela Saúde consiste em uma política de grande importância que reforça os compromissos sanitários com prioridades em saúde, definidas por gestores em suas três instâncias e enfatiza, no Pacto pela Vida, objetivos para saúde do idoso no sentido de implantar a Política Nacional de Saúde da Pessoa Idos (WANDERLEY RMM, et al., 2019). Entretanto não foi lembrada por nenhum gestor (0\%), logo, visualizá-la na prática pode ser uma tarefa difícil.

A Portaria da Secretaria de Atenção à Saúde (SAS), no1771 de 21 de novembro de 2017, reforça as avaliações dos idosos e utiliza a Caderneta de Saúde da Pessoa Idosa como guia para identificar fragilidades desta população. O uso anual da Caderneta consiste apenas emuma recomendação sugestiva e não a torna obrigatória. Portanto, compreende-se que sua utilização deve ser cada vez mais fortalecida pelos gestores (BRASIL, 2017). 
Segundo Ramos LV, et al. (2019), a Caderneta de Saúde da Pessoa Idosa auxilia na realização de ações para prestar um cuidado integral ao idoso. Além disso, é primordial que os profissionais que prestam o cuidado possuam conhecimento, por meio da educação continuada e manuais que amparem o processo de trabalho na Atenção Básica e nos diferentes níveis de atenção.

As respostas dos participantes as questões da Caderneta de Saúde do Idoso, referente a atualização do conhecimento dos profissionais e a existência dos manuais para busca do conhecimento professional foram apresentadas em números relativos (Tabela 2).

Tabela 2 - Ações de implantação da CSPI e ações de educação permanente, 2019.

\begin{tabular}{lc}
\hline Caderneta de Saúde da Pessoa Idosa & \\
\hline Implantação & Proporção \\
Sim & $100 \%$ \\
Não & - \\
\hline Período & Proporção \\
\hline 2017 & $16,6 \%$ \\
2018 & $50,0 \%$ \\
Não soube informar & $33,3 \%$ \\
\hline Processo de Implantação & Proporção \\
\hline Por meio de ações & - \\
Na campanha de vacinação de Influenza & $16,6 \%$ \\
Entregue nas consultas rotineiras da Atenção básica & - \\
Grupo Hiperdia & - \\
Outras capacitações & $83,3 \%$ \\
\hline Planejamento para a implantação & Proporção \\
\hline Sim & $100 \%$ \\
Não & - \\
\hline Existência de manual voltado para saúde do Idoso nos & Proporção \\
serviços & \\
\hline Caderneta de Saúde da Pessoa Idosa & $33,3 \%$ \\
Guia Básico de Atenção à Saúde do Idoso & $33,3 \%$ \\
Fluxo de Atendimento a Pessoa Idosa & $16,6 \%$ \\
Manual de capacitação da Caderneta de Saúde da Pessoa Idosa & $16,6 \%$ \\
\hline Capacitações realizadas na área de Saúde da Pessoa Idosa & Proporção \\
\hline 4 por ano & $50,0 \%$ \\
3 por ano & $16,6 \%$ \\
Não soube dizer & $16,6 \%$ \\
Não houve & $16,6 \%$ \\
\hline
\end{tabular}

Fonte: VIEIRA NRS, et al., 2020.

A implantação da Caderneta foi de $100 \%$ nos municípios estudados, porém houve variações quanto ao período de implantação, na qual foi observado um trabalho mais intensificado no ano de 2018 (50\%). A CSPI teve o início da sua distribuição como primeira versão no período de 2007 a 2008 nos estados e municípios. De acordo com Paulino LF, et al. (2017), após a disponibilidade da primeira versão da caderneta houve somente a intensificação de sua implantação. Assim, nota-se que ainda há muito trabalho para ser desenvolvimento nesse aspecto, visto que ainda existem municípios sem a prática do uso da caderneta.

No processo de implantação da caderneta, o método mais utilizado foram as capacitações $(83,3 \%)$, bem como a campanha de vacinação de Influenza (16,6\%), que também ajudou a disseminar e alertar sobre a importância do uso da CSPI. A quantidade de oficinas e processos de educação continuada por ano nos municípios estão na média de quatro ações $(50 \%)$. Nos serviços, existem manuais que guiam a prática assistencial, a exemplo da CSPI (33,3\%) e do Guia Básico de Atenção à Saúde do Idoso (33,3\%), confeccionado pelo município de Natal com seus parceiros.

A capacitação é o início das etapas do procedimento de implantação e se faz necessário esquematizar como será a entrega e o uso da CSPI, que pode ser realizada no momento de uma ação, em consultas 
rotineiras e até em grupos fortalecidos nas Unidades Básicas de Saúde. É importante ressaltar que, no documento que norteia o Pacto pela Vida, o cuidado à pessoa idosa é uma das metas e compromissos pactuados para melhoria da qualidade da saúde da população. As diretrizes contemplam a formação e educação continuada como pilares para a garantia de uma boa assistência (GUEDES MBOG, et al., 2017).

O processo de educação continuada e permanente visa contribuir na formação dos profissionais de saúde, conferindo aumento da autonomia e organização do processo de trabalho. Pode ser pautada em diversas metodologias, sendo as tradicionais as mais utilizadas, pois são baseadas na acumulação de informações técnico-cientificas construídas por informações e com as experiências no campo da atuação profissional. Nesta perspectiva, Ribeiro BCO, et al. (2019) e Gonçalves CB, et al. (2019) afirmam que a Política Nacional de Educação Permanente em Saúde promoveu avanços na área de educação em saúde, porém ainda são necessárias articulações para parcerias entre o serviço, ensino, educação e trabalho de forma organizada e compartilhada.

Quanto aos serviços, algumas questões foram consideradas, como acessibilidade, prioridade no atendimento e acompanhamento em serviço de atenção domiciliar (Tabela 3).

Tabela 3 - Serviços e cobertura do SAD no município, 2019.

\begin{tabular}{lc}
\hline Unidades com acessibilidade & Proporção \\
\hline Sim & $100 \%$ \\
Não & Proporção \\
\hline Quantas possuem acessibilidade & $33,3 \%$ \\
\hline 10 a 20 & $33,3 \%$ \\
20 a 30 & $16,6 \%$ \\
30 a 40 & $16,6 \%$ \\
Não soube informar & Proporção \\
\hline Serviços que atendem idosos no município & $5,2 \%$ \\
\hline Toda a rede de atenção à saúde & $15,7 \%$ \\
Atenção Básica & $10,5 \%$ \\
Rede de Urgência e Emergência & $10,5 \%$ \\
Centro de Convivência do Idoso & $10,5 \%$ \\
Centro de Especialidades Médicas & $5,2 \%$ \\
Telecentro & $10,5 \%$ \\
Centro de Referência para Saúde do Idoso & $5,2 \%$ \\
Programas dos municípios & Proporção \\
\hline Serviços que idoso possui prioridade no atendimento & $100 \%$ \\
\hline Todos da rede & - \\
Não soube informar & Proporção \\
\hline Existência de programa de atenção domiciliar & $66,6 \%$ \\
\hline Sim & $33,3 \%$ \\
Não & Proporção \\
\hline Quantos idosos são acompanhados & $16,6 \%$ \\
\hline 110 idosos & $50.0 \%$ \\
Não soube informar & $33,3 \%$ \\
Não há o serviço &
\end{tabular}

Fonte: VIEIRA NRS, et al., 2020.

A acessibilidade das unidades foi quantificada em: 10 a 20 (33,3\%) e 20 a (33,3\%). Em todos os serviços dos municípios estudados, o idoso possui prioridade de atendimento, mas em apenas um município (16,6\%) houve a inexistência de Serviço de Atenção Domiciliar (SAD). De acordo com a Constituição Federal, o direito de ir e vir deve ser assegurado a todos os cidadãos e as barreiras físicas devem ser eliminadas para reduzir a dificuldade de acesso aos serviços das pessoas que possuem mobilidade reduzida. De forma semelhante, 
Freire Junior RC, et al. (2013), aponta que o Estatuto do Idoso reforça que os locais públicos e espaços comunitários devem ser sinalizados, identificados e sem obstáculos com o objetivo de promover o acesso facilitado.

A lei no 10.048/00 garante que as pessoas vivendo com deficiência, idosos, gestantes, lactantes e pessoas acompanhadas com criança de colo terão atendimento prioritário nos locais públicos e privados, nesse sentido, todos os municípios exercem a lei de forma fidedigna (BRASIL, 2000). O SAD é direcionado para usuários do SUS que possuem dificuldades ou impossibilidades de deslocamento para o atendimento em saúde. Grande parte desses usuários necessita com maior frequência do cuidado e de serviços da rede de atenção, consequentemente uma capacidade maior do que a rede de atenção básica pode oferecer. Para Savassi LCM, et al. (2016), a atenção domiciliar promove um conjunto de ações de promoção à saúde, prevenção de doenças, tratamento e reabilitação desenvolvidas em domicílio, para garantir um cuidado integral.

Os municípios possuem avanços quanto aos programas, serviços e espaços de convivência voltados à pessoa idosa. Destes exemplos tem-se o Macaíba na Medida, o Centro Especializado em Atenção à Saúde do Idoso (CEASI), Centro de Convivência do Idoso e o Programa de Promoção ao Envelhecimento e Vida Ativa (PRO-EVA). É importante destacar a adesão e a implantação das CSPI nos municípios. Além disso, as oficinas realizadas e os parceiros na construção do conhecimento como a universidade que possibilita a realização de projetos e desempenham papéis importantes na sociedade, na qual, destaca-se o compartilhamento de saberes para a melhoria da qualidade de vida da sociedade.

\section{CONCLUSÃO}

A percepção dos gestores, quanto às políticas de saúde voltadas à pessoa idosa, aponta para familiaridade com as portarias, documentos e práticas que norteiam o cuidado no envelhecimento. No entanto, é necessário exercitar aspectos operacionais para o incremento em ações de promoção a saúde por meio de investimentos em políticas públicas de saúde eficientes, juntamente com a equipe multiprofissional e a promoção da intersetorialidade. Por fim, conclui-se que os gestores são atores principais e fundamentais para o processo de mudança do modo de produção de saúde. Por este motivo, devem ser estimulados a instituir meios para modificação dos processos de saúde e doença, contribuindo para um objetivo em comum na produção de melhorias na condição de vida da sociedade em todos os ciclos de vida.

\section{REFERÊNCIAS}

1. BALIEIRO VSL, et al. Use of the older chair as a strategy for Nursing Consultation: An experience report. Braz $\mathrm{J}$ of Develop, 2020; 6(5):1-19.

2. BRASIL. Ministério da Saúde/Secretaria de Atenção à Saúde (SAS). Portaria № 1.771, de 21 de novembro de 2017. Define a necessidades de avaliação multidimensional da saúde da pessoa idosa. Diário Oficial da União. Brasília: ministério da Saúde; 2017.

3. BRASIL. Ministério da Saúde. Pactos pela Vida, em Defesa do SUS e de Gestão. Brasília: Ministério da Saúde; 2006.

4. BRASIL. Presidência da República. Lei no 10.048 , de 8 de novembro de 2000. Dá prioridade de atendimento às pessoas que especifica, e dá outras providências. Diário Oficial da União. Brasília: Ministério da Saúde; 2000.

5. CAMARGO SM, et al. Men Care in the Aging Process: health professionals' formation. Rev Bras Em Promoção da Saúde, 2016; 29.

6. CASTRO JLC, et al. Psychosocial Analysis of Aging in the Elderly: Its Social Representations. Rev Actualidades en Psicología, 2020; 34(128):1-15.

7. DAMASCENO CKCS, et al. The Nursing Managerial Work: Professional Nurses Knowledge About Their Skills Management. Rev enferm UFPE online, 2016; 10(4):1216-1222.

8. FERREIRA VHS, et al. Ageism, Public Policies for the Elderly and Social Participation. Revista Eletrônica Acervo Saúde, 2020, 42:e2816.

9. FREIRE JUNIOR RC, et al. Estudo da acessibilidade de idosos ao centro da cidade de Caratinga, MG. Rev bras geriatr gerontol, Rio de Janeiro, 2013; 16(3):541-558.

10. GOMES CBS, et al. Política Nacional de Atenção Básica de 2017: análise da composição das equips e cobertura nacional da Saúde da Família. Rev Ciênc Saúde Coletiva, 2020; 25(4):1327-1338.

11. GONÇALVES CB, et al. The resumption of the implementation process of the National Permanent Health Education Policy in Brazil. Rev Saúde debate, 2019; 43(spe1):12-23.

REAS/EJCH | Vol.12(11) | e4948 | DOI: https://doi.org/10.25248/reas.e4948.2020 Página 8 de 9 
12. GUEDES MBOG, et al. Apoio social e o cuidado integral à saúde do idoso. Physis, Rio de Janeiro, 2017; 27(4):11851204.

13. MENDES JLV, et al. The increase of the Elderly Population in Brazil and Aging in the Last Decades: A Review of the Literature. Rev. Educ. MeioAmb. Sau., 2018; 8(1):13-26.

14. MIRANDA EC, RIVA LC. O direito dos Idosos: Constituição Federal de 1988 e Estatuto do Idoso. Rev ScienCult, $2014 ; 5(2): 125-138$.

15. MOURA LKB, et al. Bibliometric Analysis of the Scientific Evidence on Violence Perpetrated Against the Elderly. Rev Ciênc Saúde Coletiva, 2020; 25(6).

16. OHIRA RHK, et al. Profile of Primary Health Care managers in small municipalities in the north of the state of Paraná, Brazil. Ciência\&SaúdeColetiva, 2014; 19(2): 393-400.

17. PAULINO LF, et al. Elderly Subjectivation in Materials on Health Education/Communication: An Analysis from a Foucauldian Perspective. Rev. Saúde Soc., 2017; 26(4): 943-957.

18. PEREIRA KCR, et al. Evaluation of Municipal Administration of Healthcare Actions for the Elderly. Cad. SaúdePública, 2017; 33(4).

19. PIUVEZAM, G, et al. Primary Health Care and the institutionalized elderly: The perspective of municipal management in Brazil. Rev Portuguesa de Saúde Pública, 2016; 34(1):92-100.

20. RAMOS LV, et al. Use of the Elderly Person's Health Handbook in Primary Care: An Integrative Revision. Rev. Humanidades e Inovação, 2019; 6(2):272-280.

21. RIBEIRO BCO, et al. The Importance of Continuing Education and Permanent Education in the Intensive Care Unit Literature Review. Rev. Inic Cient e Ext., 2019; 2(3):167-175.

22. SAVASSI LCM, et al. The Current Challenges of Home Care in Primary Health Care: Na Analysis in the National Health System Perspective. Rev. Bras. Med. Fam. Comunidade, 2016; 11(38):1-12.

23. SILVA JLBV, et al. Health Promotion Practices for Elderly People: An Integrative Literature Review. Rev. Pesqui (Univ. Fed. Estado Rio J., Online), 2020; 12:87-93.

24. TREVISO P, et al. Competências do enfermeiro na gestão do cuidado. Rev Adm Saúde. 2017;17(69).

25. WANDERLEY RMM, et al. Evaluation of the Health Condition of the Elderly Person in Primary Care. Rev. enferm UFPE online, 2019; 13(1):472-482. 